\title{
Many-body self-localization in a translation-invariant Hamiltonian
}

\author{
Rubem Mondaini ${ }^{1, *}$ and $\mathrm{Zi} \mathrm{Cai}{ }^{2, \dagger}$ \\ ${ }^{1}$ Beijing Computational Science Research Center, Beijing 100193, China \\ ${ }^{2}$ Key Laboratory of Artificial Structures and Quantum Control, \\ Department of Physics and Astronomy, Shanghai Jiao Tong University, Shanghai 200240, China
}

\begin{abstract}
We study the statistical and dynamical aspects of a translation-invariant Hamiltonian, without quench disorder, as an example of the manifestation of the phenomenon of many-body localization. This is characterized by the breakdown of thermalization and by information preservation of initial preparations at long times. To realize this, we use quasi-periodic long-range interactions, which are now achievable in high-finesse cavity experiments, to find evidence suggestive of a divergent timescale in which charge inhomogeneities in the initial state survive asymptotically. This is reminiscent of a glassy behavior, which appears in the ground state of this system, being also present at infinite temperatures.
\end{abstract}

PACS numbers: 05.30.-d 05.45.Mt 05.70.Ln

\section{INTRODUCTION}

For a quantum particle moving in a disordered medium, the coherent backscattering from randomly distributed impurities may localize all the quantum eigenstates of the system and give rise to insulating behavior. This phenomenon, known as Anderson localization [1], is to be distinguished from another localization mechanism driven by a strong mutual repulsion of particles instead of disorder, namely, Mott localization [2,3]. Recently, it was discovered that even in the presence of small interactions Anderson localization is robust [4-8]; this was then numerically verified for a variety of quantum systems [9-28]. This quantum many-body phenomenon, dubbed many-body localization (MBL), highlights the rich physics that arises from the competition between disorder and interactions, which ultimately results in the breakdown of ergodicity and the absence of transport, even at finite energy densities, when disorder is sufficiently large.

An outstanding question is whether (many-body) localization can arise in translation-invariant systems in the absence of disorder[29-39]. The search of the disorder free localization can be traced back to Kagan and Maksimov's work on Helium mixtures consisting of two species of particles, light and heavy, where the inter-species interactions make heavy particles generate an effective random quasistatic potential which blocks the diffusion of light ones, thus localizing them [40,41]. However, recent studies suggest that such a system exhibits only transient subdiffusive dynamics, while its long-time dynamics is still ergodic, coining the term quasi-MBL to describe them $[37,39]$. A recent intriguing proposal of disorderfree localization involves an exactly solvable spin-fermion model with an extensive number of conserved quantities [42] that breaks ergodicity. In this paper, we propose

\footnotetext{
*rmondaini@csrc.ac.cn

$\dagger$ zcai@sjtu.edu.cn
}

a different approach to realize the disorder-free (manybody) localization: we study a uniform system composed of single-species identical particles, where interactions between a quantum particle and the others effectively serve as a spontaneously emergent disorder that in turn localizes the particle itself. The essential ingredient here is the inter-particle interaction with a peculiar long-range nature, which gives rise to a glassy behavior of the groundstate as well as glassy dynamics at finite energy densities.

Quantum many-body systems with long-range interactions in atomic, molecular, and optical systems have attracted considerable interest. Typical examples include dipole-dipole interactions between atoms or molecules with large dipolar momentum [43-45], Van der Waals interactions between atoms in Rydberg state [46, 47], and variable-range interactions between ultra-cold atoms in high-finesse cavities [48-50] or trapped ions [51, 52]. Compared to the cases of short-range interacting models, the localization phenomena are much less explored in long-range quantum lattice systems. It is well-known that the (Anderson) localization phenomenon strongly depends on the dimension of the system [53], thus it is natural to expect that novel localization behavior may emerge in long-range quantum lattice systems whose dimension may be not well-defined.

\section{MODEL}

We consider a one-dimensional (1D) hard-core bosonic model with infinite long-range interactions whose Hamiltonian reads,

$\hat{H}=-J \sum_{i}\left(\hat{a}_{i}^{\dagger} \hat{a}_{i+1}+h . c\right)-\sum_{i<j} V_{i j}\left(\hat{n}_{i}-\frac{1}{2}\right)\left(\hat{n}_{j}-\frac{1}{2}\right)$,

where $\hat{a}_{i}\left(\hat{a}_{i}^{\dagger}\right)$ denotes the annihilation (creation) operator for hard-core bosons, and $\hat{n}_{i}$ is the local density operator. We choose $V_{i j}=\frac{V}{L} \cos [2 \pi p|i-j|]$, where $V>0$ denotes the strength of the interactions, and $L$ is the 

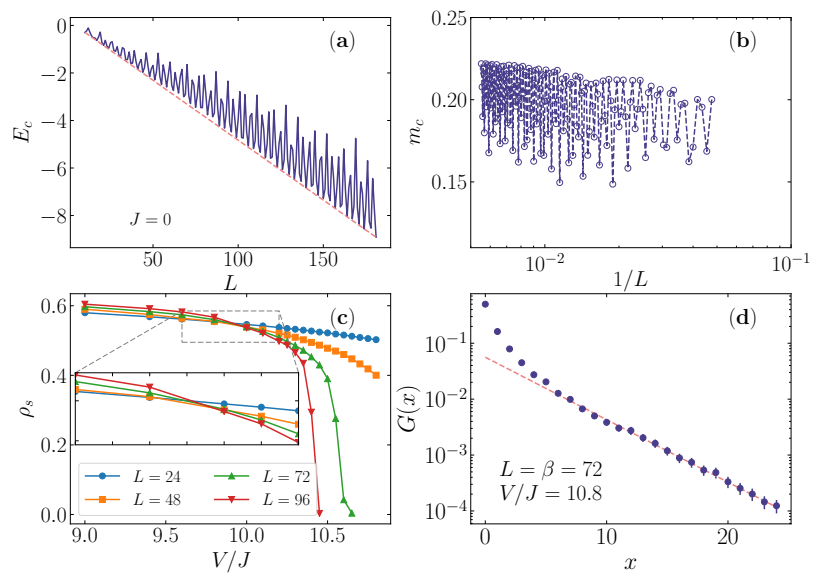

FIG. 1. (Color online) System size dependence of the total energy (a) and order parameter of the incommensurate CDW phase (b) of the classical ground state with $J=0$. (c) Superfluid density $\rho_{s}$ as a function of $V$ for different lattice sizes $L$ and $\beta(L / \beta=1)$; the inset is a zoom-in near the phase transition point. (d) The single-particle correlation function $G(x)$ in the strongly coupling phase for a system with $L=\beta=72$ and $V=10.8 \mathrm{~J}$, highlighting an exponential decay (dashed line) at large separations.

length of the $1 \mathrm{D}$ lattice. The $1 / L$ factor in the interaction term guarantees that the total interacting energy in the ground state linearly scales with the system size for sufficiently large $L$. The long-range interaction is periodic, $V_{i j}=V_{i+T, j}$, with $T=1 / p$ being the period of the interactions that can be either commensurate or incommensurate with the lattice constant $a_{0}$, which is set to unit. A key observation is that the long-range interaction is translation invariant, $V_{i j}=V_{|i-j|}$, so is the total Hamiltonian(1). We work at half-filling throughout the paper.

\section{GLASSY GROUND-STATE}

The ground state of the system with a commensurate interaction $(p=1 / 2)$ has been investigated experimentally [50] and numerically [54-58], and a rich phase diagram, including a supersolid phase, has been explored. Here, we focus on a typical incommensurate case, e.g., $p=1 / \sqrt{2}$. To gain some insight, we first study the ground state in the limit $J=0$, where the problem becomes a classical spin glass problem whose ground state energy can be estimated by the classical simulated annealing method. The total ground-state energy $E_{\mathrm{c}}$ of the Hamiltonian (1) in this limit $(J=0)$ as a function of lattice length is plotted in Fig. 1(a), from which we find that, even though $E_{\mathrm{c}}$ dramatically oscillates with $L$, as a consequence of the incommensurate infinite long-range interaction, the lower enveloping line of the $E_{c}-L$ curve linearly depends on $L$, indicating that in the thermodynamic limit, the average energy per site is independent of the system size. To deter- mine the nature of this classical ground state, we introduce an order parameter to measure the incommensurate charge density wave (CDW) phase with period $1 / p$, $m_{c} \equiv \frac{1}{L} \sqrt{\sum_{i, j}\left(n_{i}-\frac{1}{2}\right)\left(n_{j}-\frac{1}{2}\right) e^{\imath 2 \pi p(i-j)}}$. As shown in Fig. 1(b), when $L \rightarrow \infty, m_{c}$ extrapolates to a finite value, indicating a long-range CDW correlation in the classical ground state. Now, we turn on the hopping in the above classical picture, which plays a role in inducing quantum fluctuations. To study the ground state of this model, we perform quantum Monte Carlo (QMC) simulations with a worm algorithm update [59-61], which is free from the sign problem since the frustration appears only in the diagonal (interacting) part of $\hat{H}$.

To determine the properties of the ground state, we calculate the superfluid density $\rho_{s}$ (computed using the winding number $W$ [62] of the QMC simulations as $\left.\rho_{s} \equiv(L / \beta)\left\langle W^{2}\right\rangle\right)$ and perform the finite size scaling with the dynamical critical exponent $z=1$, with the scaling relation between the space and imaginary time as $L / \beta=1$. As shown in the classical case, the physical quantities in the ground state may dramatically oscillate with the lattice length, thus special attention needs to be taken for the finite size scaling. To derive the properties in the thermodynamic limit, we choose lattices whose length is close to the lower enveloping line of the $E_{c}-L$ curve. The superfluid density $\rho_{s}$ as a function of interaction strength $V$ is plotted in Fig. 1(c), from which we find that for sufficiently large $V, \rho_{s}$ vanishes, indicating the breakdown of the quasi-superfluidity. In the inset of Fig. 1(c), we obtain a scaling invariant point at $V_{c}=9.9 \pm 0.1 J$, which indicates a continuous phase transition with dynamical critical exponent $z=1$, separating a Luttinger liquid and a strongly interacting phase.

Now, we analyze the nature of the strongly interacting regime, which should adiabatically connect to the classical limit $J=0$. In Fig. 1(d), we plot the equaltime single-particle correlation function $G(x)=\left\langle\hat{a}_{i}^{\dagger} \hat{a}_{i+x}\right\rangle$, which decays exponentially in distance. Inspired by the results in the classical limit, we can introduce the incommensurate CDW order parameter $m_{c}$ and decouple the interaction under mean-field approximation in the thermodynamic limit and the Hamiltonian can be rewritten as

$H_{\mathrm{MF}}=-J \sum_{i}\left(a_{i}^{\dagger} a_{i+1}+\right.$ h.c. $)-2 V m_{c} \cos (2 \pi p i) n_{i}+V m_{c}^{2}$

with $p=1 / \sqrt{2}$. Hence, this mean-field Hamiltonian is equivalent to the Aubry-André model (See Appendix A), which is known to have a phase transition when increasing the strength of the incommensurate external potential [63]. However, in our case, the translational symmetry breaks spontaneously instead of explicitly.

Glassy behavior in the absence of disorder also appears in other contexts of systems with long-range interactions either in a lattice [65] or in the continuous [66]. 

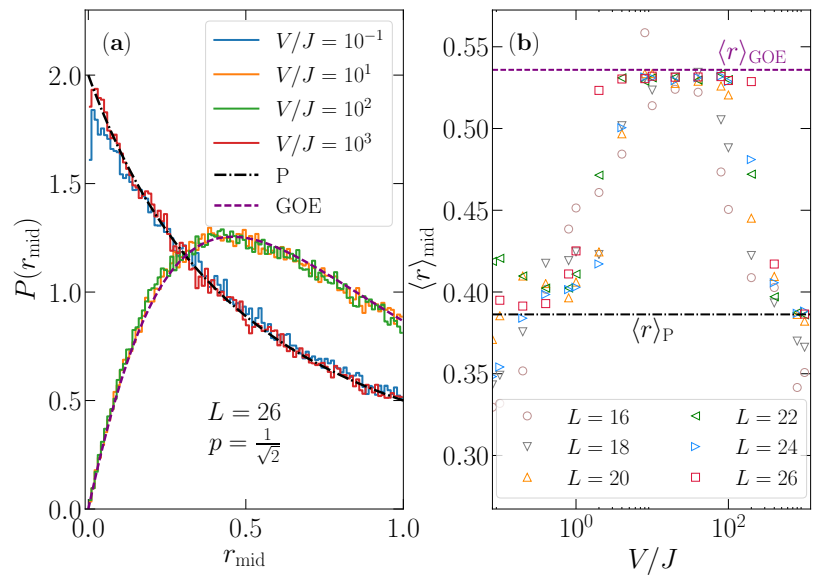

FIG. 2. (Color online) Statistics of adjacent gaps probing ergodicity/non-ergodicity. (a) The distribution of the ratio of adjacent gaps in the central half of the spectrum $(L=26)$ and different magnitudes of the interactions. The dashed (dasheddotted) line depicts the analytical expression of an ergodic (nonergodic) quantum system described by GOE (Poisson) statistics. (b) The average value of this quantity as $V$ is varied: for small and large values, nonergodic behavior sets in, while for intermediate interactions, quantum chaos prediction is obtained. To decrease statistical fluctuations, all values are averaged between the equivalent parity sectors of the system's symmetries (see text). Dashed lines describe the ergodic (nonergodic) regimes with $\langle r\rangle \approx 0.5359$ (0.3863) [64].

\section{FINITE ENERGY DENSITIES: STATISTICAL PROPERTIES}

It is an open question whether localization present in the ground state of a system could be connected to the localization phenomenon in highly excited states. In our model, specifically, we aim to verify if the previously described glasslike phase also survives at finite energy densities. We begin with a diagnostic of quantum chaotic behavior [67-69], which has been widely used as a way to probe ergodicity in quantum systems. This can be quantified by the presence or not of energy level repulsion [70-72], which in turn, can be measured via the ratio of adjacent gaps in the spectrum [9, 64], $r_{\alpha} \equiv$ $\min \left(\delta_{\alpha+1}, \delta_{\alpha}\right) / \max \left(\delta_{\alpha+1}, \delta_{\alpha}\right)$, and $\delta_{\alpha}=E_{\alpha}-E_{\alpha-1}$ are gaps in between consecutive energy levels in the ordered list of eigenenergies $\left\{E_{\alpha}\right\}$ of the Hamiltonian. We show in Fig. 2(a) the distribution of $r$ in the central half of the spectrum[73], averaged among all the real sectors of the Hamiltonian, obtained by using a basis that encodes translation, particle-hole, and inversion symmetries (See Appendix B for a description of the symmetry resolving), for the largest system size we study, $L=26$ (Hilbert space dimension $\mathcal{D} \sim 10^{5}$ ). In the limit $V / J \ll 1$, the system is close to the integrable regime and level repulsion is absent: the level spacings are completely uncorrelated and a Poisson distribution is obtained [64]. On the other hand, for increasing interactions, the distribution becomes equivalent to the ones of symmetric ran- dom matrices belonging to a Gaussian orthogonal ensemble (GOE) [64], which is characteristic of thermalizing quantum systems. Nevertheless, when approaching a regime where the interaction strength is much larger than the hopping scale, a Poisson distribution for $r$ is once again recovered. This suggests that localization, described by the breakdown of ergodicity, at infinite temperatures is obtained in a manner similar to the scenario of MBL for large enough quenched disorder, but here in a translation-invariant system.

The Hamiltonian (1) is nonintegrable for any finite value of the interactions so one would expect the predictions of the eigenstate thermalization hypothesis (ETH) $[67-69,74]$ to be numerically obtained, apart from finite size effects [75]. To rule these out, we check in Fig. 2(b) the average value of these distributions for different system lattice sizes and interactions. The intermediate ergodic regime $\left(\mathrm{V} / \mathrm{J} \sim 10^{1}-10^{2}\right)$ is robust and level repulsion, characterized by $r \sim r_{\mathrm{GOE}}$, is absent for $V / J \gtrsim 10^{2}$. As for the ground state, a proper finite-size scaling is rather elusive [76].

\section{DYNAMICAL LOCALIZATION}

A clearer picture of whether a transition from thermalization to MBL-like behavior takes place, at large interaction values, can be quantified in the dynamical properties of the system. We start by investigating the dynamical inverse participation ratio [35, 36] (IPR), which is a measure of localization in the many-body basis, $\mathcal{I}_{\boldsymbol{j}}(\tau)=\mathcal{D}^{-1}\left[\sum_{\boldsymbol{i}}\left|\left\langle\psi_{\boldsymbol{j}}(\tau) \mid \boldsymbol{i}\right\rangle\right|^{4}\right]^{-1}$, obtained after time evolving an initial state $|\boldsymbol{j}\rangle\left(\left|\psi_{\boldsymbol{j}}(\tau)\right\rangle=e^{-\imath \hat{H} \tau}|\boldsymbol{j}\rangle\right)$ in the fully symmetric basis of $\hat{H}$. To reduce statistical effects, we average over all initial states in each sector as well as the results from different (real) symmetric ones, obtaining $\langle\overline{\mathcal{I}}(\tau)\rangle$. At long times, a time-evolved state is localized [delocalized] in the basis if the dynamical IPR is proportional to $O\left(\mathcal{D}^{-1}\right)[O(1)]$. Figure 3(a) shows that with increasing interactions, the equilibrium value of the dynamical IPR is substantially reduced and the system may fully retain information about its initial preparation at large time-scales. To account for finite size effects, we compare the equilibrium value $\langle\overline{\mathcal{I}}(\tau)\rangle_{\text {eq. }}$. of the dynamical IPR for different lattices in Fig. 3 (b). A crossing of the curves is obtained for values of $V / J$ in the range $\sim 130-680$ [see inset in Fig. 3(b)], after which one expects that localization survives in the thermodynamic limit, defining the critical value of interactions where the MBL-like behavior takes place and thermalization no longer holds.

\section{INITIAL STATE INHOMOGENEITIES}

We further characterize the interaction-induced quantum glass transition by noting how an inhomogeneity in the particle distribution in real space for the initial 

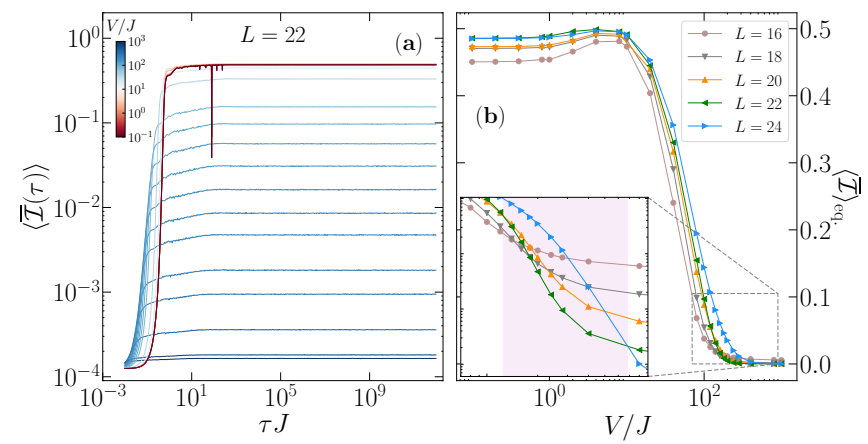

FIG. 3. (Color online) (a) Time dependence of the dynamical IPR for a lattice with $L=22$ averaged over all equivalent real parity sectors, and different initial states, for several interaction strengths given by the color code. Panel (b) shows its equilibrium value in the long-time limit $\langle\overline{\mathcal{I}}\rangle_{\text {eq. }} \equiv\langle\overline{\mathcal{I}}(\tau \rightarrow \infty)\rangle$ as a function of $V / J$, comparing the size dependence. The inset highlights the crossing region marking the beginning of the MBL-like regime; the shaded area represents the confidence interval for the critical interaction.

state persists after the unitary time-evolution $[29,34,36]$. This has the advantage of being relevant to experiments in optical lattices that can probe site-resolved densities. We define the charge inhomogeneity operator $(\Delta \hat{n})^{2} \equiv(1 / L) \sum_{i}\left(\hat{n}_{i+1}-\hat{n}_{i}\right)^{2}$, whose expectation values within the initial symmetric Fock states are in the range $\left[\frac{2}{L}, \frac{4}{L}, \ldots, 1\right]$, quantifying the number of domain walls $\left(N_{\mathrm{dw}}\right)$ present in these states. Figures 4 (a) and 4(b) display the time-integrated charge inhomogeneity, $\overline{(\Delta n)^{2}}(\tau)=(1 / \tau) \int_{0}^{\tau}(\Delta n)^{2}\left(\tau^{\prime}\right) d \tau^{\prime}$, in the thermal [Fig. $4\left(\right.$ a) $-V / J=10^{1}$ ] and nonergodic regimes [Fig. 4(b) - $V / J=10^{3}$, averaged over all the initial states with equivalent $N_{\mathrm{dw}}$. The differences are clear: in the former, the time evolution results in a featureless state irrespective of the value of $(\Delta n)^{2}(0)$, while in the latter the charge inhomogeneity of the initial state is preserved for arbitrarily long times. Appendix E shows that this is intrinsically related to the incommensurability of the interactions with respect to the lattice spacings by checking other values of periodicities $p$. In contrast, if one considers commensurate interactions as $p=1 / 2$, information about the initial preparation is eventually lost as shown in Appendix F. Figures 4(c) and 4(d) compare the infinite-time average, given by the diagonal ensemble (DE), for all the initial symmetric Fock states with the corresponding microcanonical prediction [69, 74] showing the breakdown of thermalization in the strongly interacting regime. Appendix $\mathrm{C}$ displays similar results for a larger lattice size, $L=26$.

\section{MANY-BODY DENSITY OF STATES AND ETH}

When investigating a translation-invariant MBL system, one needs to be careful with the possible formation of "mini-bands" in the spectrum of finite systems [37].

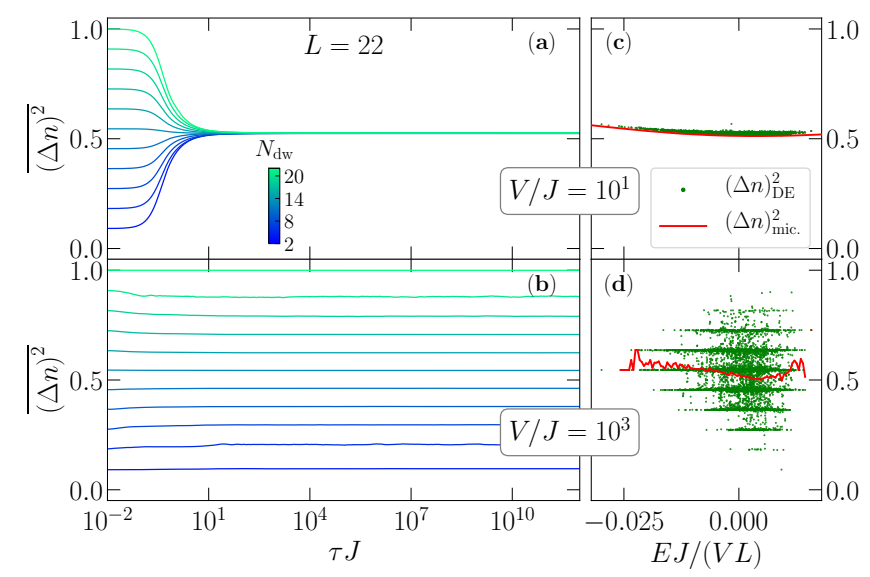

FIG. 4. (Color online) Time evolution of the integrated charge inhomogeneity $\overline{(\Delta n)^{2}}$ averaged over states with similar $N_{\mathrm{dw}}$ in ergodic [nonergodic] regimes in panel (a)[panel (b)] with $V / J=10^{1}\left[V / J=10^{3}\right]$. Panels (c) and (d) are comparisons of the DE ensemble prediction and the microcanonical result for the corresponding values of interactions.

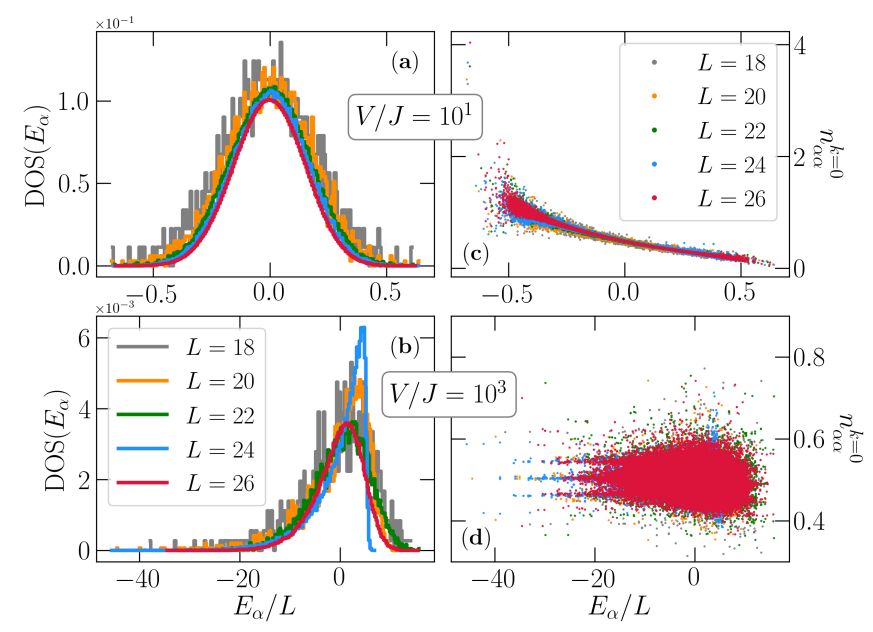

FIG. 5. (Color online) Normalized density of states [panels (a) and (b)] and eigenstate expectation values of the zeromomentum occupation $n_{\alpha \alpha}^{k=0}$ [panels (c) and (d)] for $V / J=10$ [panels (a) and (c)] and $V / J=10^{3}$ in [panels (b) and (d)]. The clear ETH-like behavior for $n_{\alpha \alpha}^{k=0}$ in panel (c) is lost once interactions are further strengthened (d).

These are typical in proposals of translation-invariant MBL consisting of two types of particles (light and heavy) $[37,39]$ with very different energy scales; they reduce the effective Hilbert space and do not persist in the thermodynamic limit, resulting in pseudo-MBL behavior that is essentially a finite size effect. To test this scenario, Fig. 5 (a) and 5(b) show the normalized density of states (DOS) with increasing magnitudes of interactions. Albeit some rather large finite-size effects affect the distribution shape (see, e.g., $L=24$ results and Appendix D concerning the monotonicity of thermodynamic quantities), the DOS does not display a separation into small bands for the case of a real even (under all parity symmetries) 
sector of the Hamiltonian, even at the largest interaction $V / J=10^{3}$, which already displays an MBL-like behavior for other quantities. Besides, it is clear that in this strong interaction regime, thermalization, as prescribed by the ETH, fails with the support of the eigenstate expectation values of a few-body operator, like the zero momentum occupation $\left[\hat{n}^{k=0}=(1 / L) \sum_{i, j} \hat{a}_{i}^{\dagger} \hat{a}_{j}\right]$ displayed in Figs. 5 (c) and $5(\mathrm{~d})$, not decreasing with system size.

\section{SUMMARY}

Using large-scale numerical calculations, ranging from classical annealing to QMC to exact diagonalizations, we study a quantum glass transition of a system composed of hard-core bosons subjected to quasiperiodic long-range interactions. This translation-invariant system displays a transition to localized behavior at sufficiently large interaction magnitudes not only at the groundstate but also at finite energy densities. In the latter, we associate it to an MBL-like regime where the breakdown of thermalization and initial state memory survives at exponentially large time scales, even in the absence of any disorder. Although the infinite time-average for initial states with typical values of $N_{\mathrm{dw}}$ may approach the thermal prediction in the large interaction limit, the number of (initial) states that result in a discrepancy is not necessarily rare, suggesting that initial memory survives indefinitely, even at infinite temperatures (see Appendix C), unlike in the scenario of translation-invariant MBL proposals of "light" and "heavy" particles. Nevertheless, the recent emulation of long-range interactions in optical lattices embedded in high-finesse cavity experiments, emulating the physics described here, may settle this issue by probing whether the so far numerically observed outof-equilibrium localization in systems without quenched disorder is a phenomenon that is not related to finite size effects.

\section{ACKNOWLEDGMENTS}

R.M. is financially supported by the National Natural Science Foundation of China (NSFC) (Grants No. U1530401, No. 11674021, and No. 11650110441). ZC is supported by the National Key Research and Development Program of China (Grant No. 2016YFA0302001), the National Natural Science Foundation of China under Grant No.11674221, and the Shanghai Rising-Star Program. R.M. acknowledges enlightening discussions with Deepak Iyer and Marcos Rigol. Z.C. thanks L. Pollet for valuable discussions. The computations were performed on the Tianhe-2JK at the Beijing Computational Science Research Center (CSRC). Z.C. acknowledges the support from the Center for High Performance Computing of Shanghai Jiao Tong University.

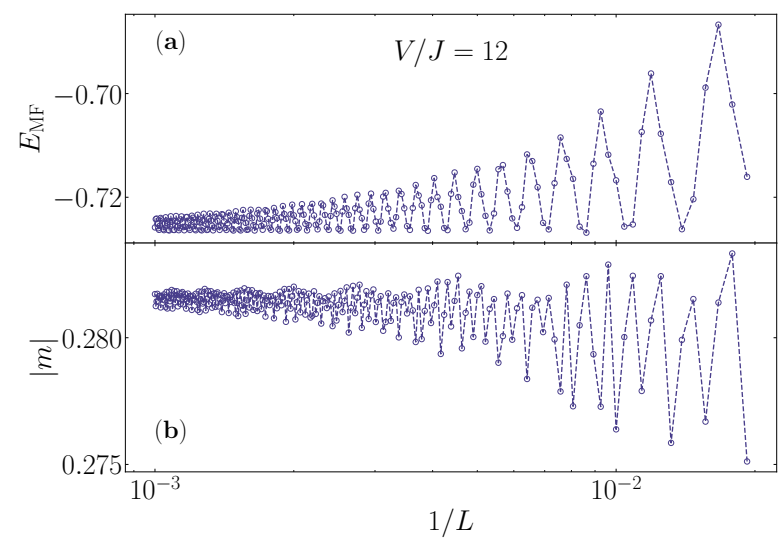

FIG. 6. (Color online) (a) System size dependence of the ground-state energy per site obtained within the mean-field approximation and (b) the CDW order parameter obtained from the mean-field for an interaction strength $\mathrm{V} / \mathrm{J}=12$ with incommensurate interactions whose inverse period is $p=$ $1 / \sqrt{2}$.

\section{Appendix A: Mean-field analysis}

We explore here in more details the mean field version of the Hamiltonian that is valid in the strongly interacting regime. The original Hamiltonian can be rewritten as

$$
\begin{aligned}
& \hat{H}=-J \sum_{i}\left(\hat{a}_{i}^{\dagger} \hat{a}_{i+1}+h . c\right)-\frac{V}{2 L}\left\{\left[\sum_{i} e^{\imath 2 \pi p i}\left(\hat{n}_{i}-\frac{1}{2}\right)\right]\right. \\
& \left.\cdot\left[\sum_{j} e^{-\imath 2 \pi p j}\left(\hat{n}_{j}-\frac{1}{2}\right)\right]+h . c\right\} .
\end{aligned}
$$

By introducing the mean-field charge-density-wave order parameter: $m=\frac{1}{L} \sum_{i} e^{-i 2 \pi p i}\left(\left\langle\hat{n}_{i}\right\rangle-\frac{1}{2}\right)$, the Hamiltonian in Eq.(A1) can be decoupled as:

$$
\begin{aligned}
\hat{H} & =\sum_{i}-J\left(\hat{a}_{i}^{\dagger} \hat{a}_{i+1}+h . c\right) \\
& -\sum_{i}\left\{V\left[m e^{-\imath 2 \pi p i}\left(\hat{n}_{i}-\frac{1}{2}\right)+h . c\right]+\frac{V}{2}|m|^{2}\right\} .(
\end{aligned}
$$

The translational symmetry has been broken explicitly in the mean-field Hamiltonian (A2), where the meanfield order parameter $m$ can be solved using the standard self-consistent method in real-space. In Fig. 6 (a) and Fig. 6 (b), we plot the result of the ground-state energy and the mean-field order parameter $|m|$ as a function of the system size $L$ for a fixed $V / J=12$, which according with the results from the QMC, is already in the strongly correlated regime. We note that the fluctuations markedly decrease for larger system sizes properly defining the thermodynamic limit. This behavior is also expected in classical case $(J=0)$, originally presented in Figs. 1 (a) and (b) in the main text, provided one is able to reach larger system sizes. 


\section{Appendix B: Symmetry sector resolving}

To probe aspects related to quantum chaotic behavior (and the lack of thereof for large enough interactions) encoded in the level repulsion, or to compute infinite timeaverages (diagonal ensemble averages), one most ensure that all "trivial" degeneracies are resolved. These are in general related to the symmetries of the Hamiltonian. In our model, these are three: translation $\left(\hat{T}_{x}\right)$, and parities under particle-hole $\left(\hat{P}_{\text {phs }}\right)$ and reflection $\left(\hat{P}_{I}\right)$. We construct a basis where the states are simultaneously eigenstates of these three symmetry operations, which reduces the total Hilbert space $\mathcal{D}=\left(\begin{array}{c}L \\ L / 2\end{array}\right)$ to smaller sectors $\mathcal{D}^{\prime} \approx \mathcal{D} /(L \cdot 2 \cdot 2)$. We restrict ourselves to real sectors (with corresponding momentum quantum numbers $k=0$ or $\pi$ ) and, to reduce statistical fluctuations, we average over the 8 equivalent sectors after the parity operations in Figs. 2 and 3. Whereas in Figs. 4 and 5, we restrict to the even sectors under $\hat{P}_{\mathrm{phs}}$ and $\hat{P}_{I}$ for the zero momentum translation quantum number.

\section{Appendix C: Infinite time average and the initial memory conservation}

The initial memory preservation is a characteristic of many-body localized systems. In Fig. 4 (main text), we show that, in the strongly interacting (non-ergodic) regime, a discrepancy is clear between the infinite time average of the charge inhomogeneities, encoded in the initial (symmetric) Fock states, and the values of a thermal ensemble, signaling the thermalization breakdown. Here, we repeat this analysis in Fig. 7 for the largest system size we study, presenting qualitatively similar results. The states for which the discrepancy is larger, are the ones that have a number of domain walls $\left(N_{\mathrm{dw}}\right)$ far from the typical value, which displays infinite time predictions closer to the thermal result. We argue here that although the number of those states is small, they still represent a substantial fraction of the total number of initial Fock states. This can be seen by the histogram in panel (c) that counts the values of the diagonal ensemble results of $(\Delta \hat{n})^{2}$ for initial states whose energies $E_{0}(=\langle\psi(0)|\hat{H}| \psi(0)\rangle)$ are within the central half of the system's eigenenergies. In the thermodynamic limit, the peak-structure will become smoother but with large tails that will manifest the lack of thermalization.

\section{Appendix D: Entropy at infinite temperatures}

One concern that may arise with the manifest large finite-size effects appearing in the strongy correlated regimes, refers to the monotonicity of thermodynamic quantities. For example, the thermodynamic entropy $S$ at energy $E$ is related to the density of states via $e^{S(E)}=E \sum_{\alpha} \delta\left(E-E_{\alpha}\right)$. At infinite temperatures, one

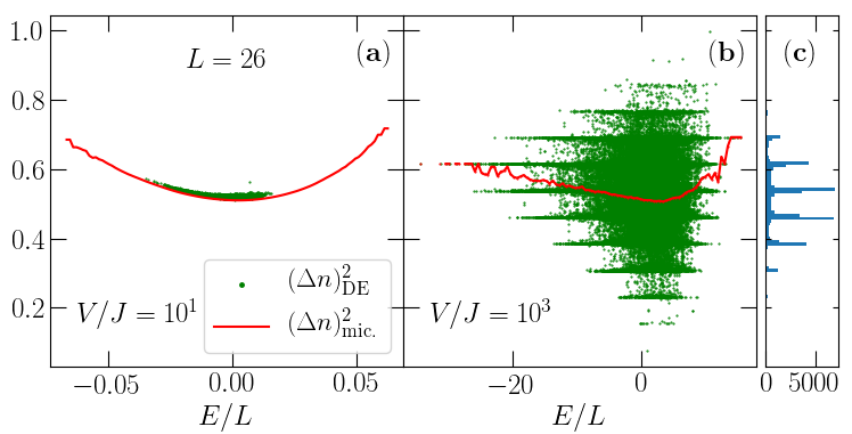

FIG. 7. (Color online) Comparisons of the infinite timeaverage (diagonal ensemble prediction) of the charge inhomogeneity $(\Delta \hat{n})^{2} \equiv(1 / L) \sum_{i}\left(\hat{n}_{i+1}-\hat{n}_{i}\right)^{2}$ and the microcanonical (thermal) results $\left.\left[(\Delta \hat{n})^{2}\right)_{\text {mic. }}=\sum_{\alpha}|\langle\alpha \mid \psi(0)\rangle|^{2}(\Delta \hat{n})_{\alpha \alpha}^{2}\right]$ in the ergodic (a) and non-ergodic (b) regimes for a lattice with 26 sites. Panel (c) depicts the histogram of the average charge inhomogeneities when $\tau \rightarrow \infty$ at infinite temperatures (see text).

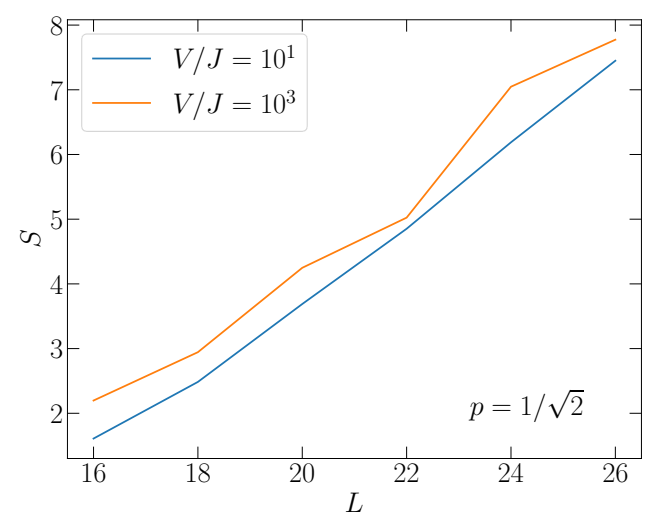

FIG. 8. (Color online) Thernodynamic entropy at infinite temperatures vs. the lattice size in the ergodic $\left(\mathrm{V} / \mathrm{J}=10^{1}\right)$ and non-ergodic regimes $\left(V / J=10^{3}\right)$ for a subsector of the Hamiltonian with zero total momentum and with even parities under particle-hole and reflection symmetries.

can write down the thermodynamic entropy as the logarithm of the density of states at its maximum value. Figure 8 displays the system size dependence of this quantity for two values of interactions, $V / J=10^{1}$ and $10^{3}$, in the ergodic and non-ergodic regimes, respectively. In the latter, the fluctuations are a direct manifestation of the finite-size effects but still show how the entropy is a momotonic function of the system size.

\section{Appendix E: Incommensurate case: other periodicities}

We have established that the system displays localizaton at infinite temperatures for the case of long-range quasi-periodic interactions with period $T=\sqrt{2}$. Nevertheless, finite size effects are strongly manifest in a variety of quantities when determining the boundary between the ergodic regime and the many-body localized 

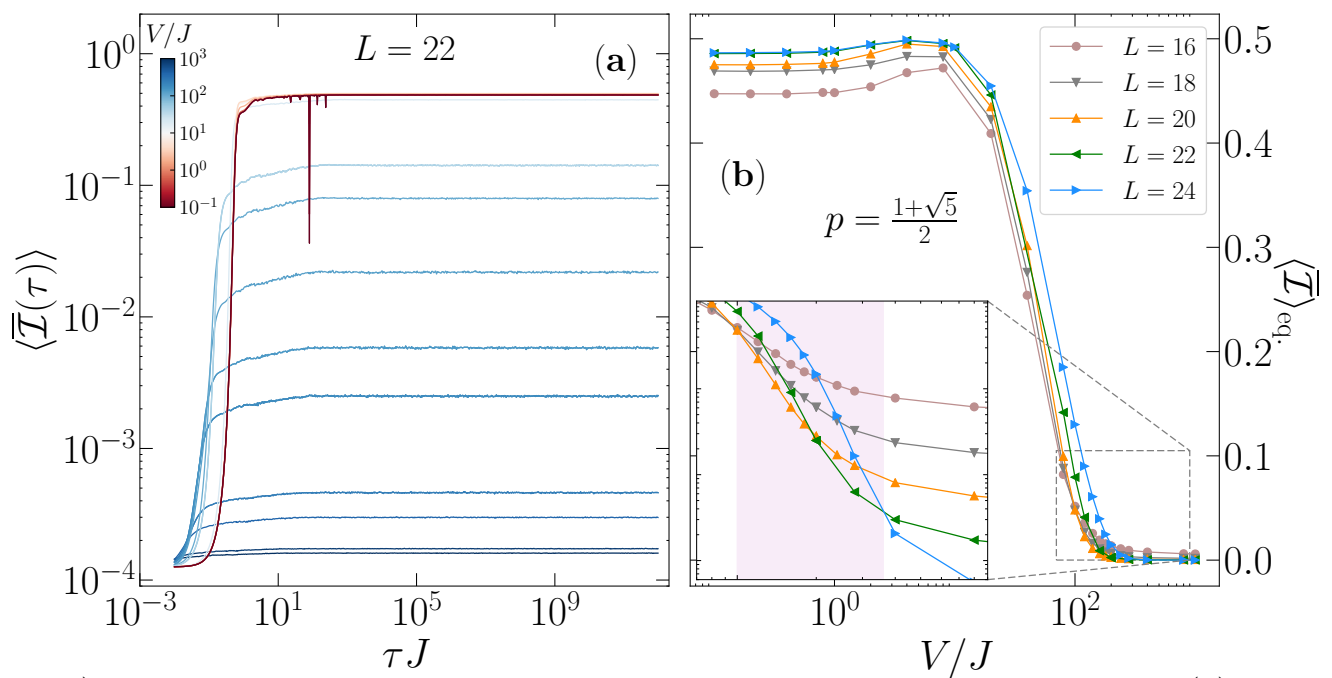

FIG. 9. (Color online) Same as in Fig. 3. The crossing region, given by the shaded area in the inset of (b), depicts the confidence interval of the localization transition given the system sizes available: $V_{c} / J=100-360$. This is consistent with the other periodicity $p=1 / \sqrt{2}$ though the interval is reduced due to smaller finite-size effects.

one. One of the quantities that is largely affected is the dynamical inverse participation ratio (Fig. 3 in the main text), where the results for system size $L=24$ fail to reach a crossing point in $V / J$ compatible with other lattice sizes. That this size presents pathological behavior can also be inferred from the non-gaussian behavior of the many-body density of states in the strongly interacting limit (Fig. 5(b) in the main text), in contrast to other values of $L$. One of the reasons behind this is that the period of interactions is almost commensurate with the lattice size for $L=24$ when $p=1 / \sqrt{2}$.

Different approaches may be used to mitigate finite size effects. We choose a simple one in which we see how robust is the transition point under variations of the incommensurate periodicity of the interactions. We select a new period $T=1 / p$ with $p$ given by the golden ratio $\frac{1+\sqrt{5}}{2}$ and report in Fig. 9 the same as in Fig. 3 of the main text but for this different periodicity. We note that for the system sizes studied for this quantity (even number of lattice sites from $L=16$ to 24 ) the crossing point (around $V_{c} / J=100-360$ ) is consistent with the previous estimation from $p=1 / \sqrt{2}$.

\section{Appendix F: The commensurate case: $p=1 / 2$}

The case of interactions whose period $T=1 / p$ is twice the lattice constant corresponds to the realistic situation in the experiment described in Ref. [50]. In this scenario, all the lattice sites can be classified into even and odd groups and the interaction between the two particles in the different(same) group are repulsive (attractive) with the same interaction strength. The interaction can be rewritten as: $\hat{H}_{\text {int }}=-\frac{V}{L}\left(\hat{N}_{e}-\hat{N}_{o}\right)^{2}$ where $\hat{N}_{e}\left(\hat{N}_{o}\right)$ is the total particle number operator in the even (odd) sublattice. Notably, the interaction favors the formation of
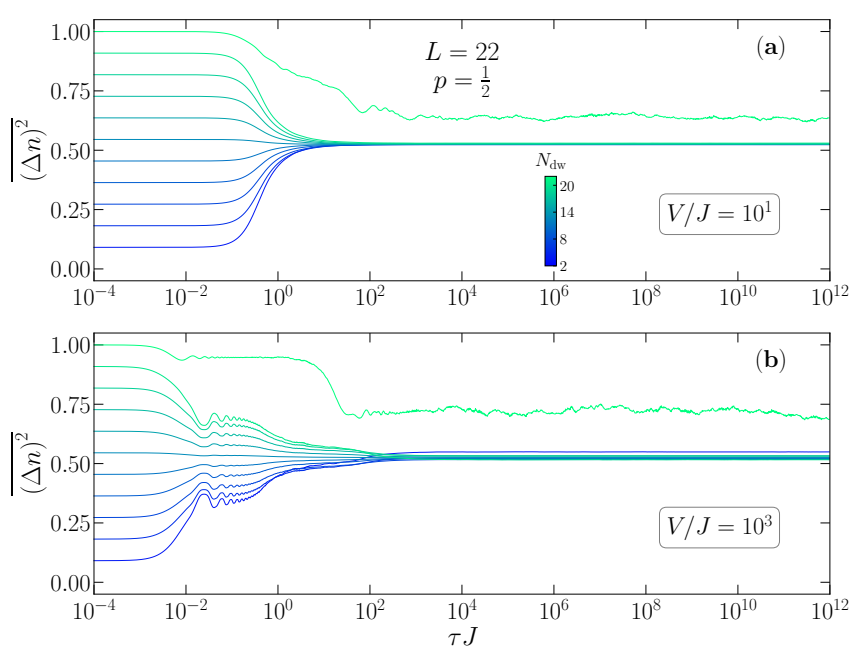

FIG. 10. (Color online) Similar to Figs. 4(a) and 4(b) in the main text but for the case of interactions whose period is commensurate with the lattice spacing $(p=1 / 2)$. The time-dependence of the integrated charge inhomogeneity is reported for interactions $V / J=10^{1}\left[10^{3}\right]$ in (a) $[(\mathrm{b})]$.

a charge-density-wave (CDW) state with a non-zero order parameter: $m=\left\langle\hat{N}_{e}-\hat{N}_{o}\right\rangle / L$. A simple mean-field treatment of the Hamiltonian suggests that even an infinitesimal $V$ will open a gap and induce a CDW insulating phase with spontaneously translational symmetry breaking.

In the out-of-equilibrium situation, to highlight that this model does not give similar conclusions as to the incommensurate case $p=1 / \sqrt{2}$ studied in the main text, we show in Fig. 10 the quantity which can be used experimentally to probe localization, the degree of charge inhomogeneity of time-evolved initial states.

For similar values of interactions which are in the er- 
godic and non-ergodic regimes for $p=1 / \sqrt{2}, V / J=10^{1}$ and $10^{3}$, respectively, we see that the initial charge inhomogeneity vanishes in both cases, resulting in a featureless state for large enough time-scales in contrast to the localization observed for large interaction values in the case of incommensurate interactions. As in the main text, we average the time-integrated charge inhomogeneity, $\overline{(\Delta n)^{2}}(\tau)$, for states with equivalent number of domain walls that quantifies the degree of inhomogeneity. The notable exception is the initial state with maximum number of domain walls $\left[\overline{(\Delta n)^{2}}(0)=1\right]$, corresponding to a CDW initial state - in fact, a symmetric version incorporating all the symmetries of the basis. This state approaches the actual ground-state of the Hamiltonian in the large $V$ limit and possess total energy that is largely gapped from the bulk of the spectrum. This results in a lack of hybridization with other states with different degrees of charge-inhomogeneity, then partially preserving information of the initial state. Yet it does not constitute though a localization feature at infinite temperatures, characteristic of many-body localization.

We note as well that in the irreducible sectors of the Hamiltonian - after applying translation, inversion and particle-hole symmetries - the spectrum does not display level repulsion in any range of the interaction magnitude. That is suggestive that the Hamiltonian might display integrability for commensurate interactions as, e.g., for $p=1 / 2$. Thus, this precludes a scenario of breakdown of ergodicity signaling the onset of translation-invariant many-body localization.
[1] P. W. Anderson, "Absence of diffusion in certain random lattices," Phys. Rev. 109, 1492 (1958).

[2] N. F. Mott, "The basis of the electron theory of metals, with special reference to the transition metals," Proceedings of the Physical Society. Section A 62, 416 (1949).

[3] N. F. Mott, "Metal-insulator transition," Rev. Mod. Phys. 40, 677 (1968).

[4] L. Fleishman and P. W. Anderson, "Interactions and the Anderson transition," Phys. Rev. B 21, 2366 (1980).

[5] B. L. Altshuler, Y. Gefen, A. Kamenev, and L. S. Levitov, "Quasiparticle lifetime in a finite system: A nonperturbative approach," Phys. Rev. Lett. 78, 2803 (1997).

[6] I. V. Gornyi, A. D. Mirlin, and D. G. Polyakov, "Interacting electrons in disordered wires: Anderson localization and low-T transport," Phys. Rev. Lett. 95, 206603 (2005).

[7] D. M. Basko, I. L. Aleiner, and B. L. Altshuler, "Metalinsulator transition in a weakly interacting many-electron system with localized single-particle states," Ann. Phys. 321, 1126 (2006).

[8] D. M. Basko, I. L. Aleiner, and B. L. Altshuler, "On the many-body localization phenomena," in Problems of Condensed Matter Physics: Quantum coherence phenomena in electron-hole and coupled matter-light systems, edited by Alexei L. Ivanov and Sergei G. Tikhodeev (Oxford University Press, 2007).

[9] V. Oganesyan and D. A. Huse, "Localization of interacting fermions at high temperature," Phys. Rev. B 75, 155111 (2007).

[10] M. Žnidarič, T. Prosen, and P. Prelovšek, "Many-body localization in the Heisenberg XXZ magnet in a random field," Phys. Rev. B 77, 064426 (2008).

[11] C. Monthus and T. Garel, "Many-body localization transition in a lattice model of interacting fermions: Statistics of renormalized hoppings in configuration space," Phys. Rev. B 81, 134202 (2010).

[12] A. Pal and D. A. Huse, "Many-body localization phase transition," Phys. Rev. B 82, 174411 (2010).

[13] E. Canovi, D. Rossini, R. Fazio, G. E. Santoro, and A. Silva, "Quantum quenches, thermalization, and manybody localization," Phys. Rev. B 83, 094431 (2011).

[14] E. Khatami, M. Rigol, A. Relaño, and A. M. GarciaGarcia, "Quantum quenches in disordered systems: Ap- proach to thermal equilibrium without a typical relaxation time," Phys. Rev. E 85, 050102(R) (2012).

[15] J. H. Bardarson, F. Pollmann, and J. E. Moore, "Unbounded growth of entanglement in models of many-body localization," Phys. Rev. Lett. 109, 017202 (2012).

[16] S. Iyer, V. Oganesyan, G. Refael, and D. A. Huse, "Many-body localization in a quasiperiodic system," Phys. Rev. B 87, 134202 (2013).

[17] M. Serbyn, Z. Papić, and D. A. Abanin, "Universal slow growth of entanglement in interacting strongly disordered systems," Phys. Rev. Lett. 110, 260601 (2013).

[18] M. Serbyn, Z. Papić, and D. A. Abanin, "Local conservation laws and the structure of the many-body localized states," Phys. Rev. Lett. 111, 127201 (2013).

[19] M. Serbyn, M. Knap, S. Gopalakrishnan, Z. Papić, N. Y. Yao, C. R. Laumann, D. A. Abanin, M. D. Lukin, and E. A. Demler, "Interferometric probes of many-body localization," Phys. Rev. Lett. 113, 147204 (2014).

[20] D. A. Huse, R. Nandkishore, and V. Oganesyan, "Phenomenology of fully many-body-localized systems," Phys. Rev. B 90, 174202 (2014).

[21] R. Nandkishore, S. Gopalakrishnan, and David A. Huse, "Spectral features of a many-body-localized system weakly coupled to a bath," Phys. Rev. B 90, 064203 (2014).

[22] D. Pekker, G. Refael, E. Altman, E. Demler, and V. Oganesyan, "Hilbert-glass transition: New universality of temperature-tuned many-body dynamical quantum criticality," Phys. Rev. X 4, 011052 (2014).

[23] A. Chandran, I. H. Kim, G. Vidal, and D. A. Abanin, "Constructing local integrals of motion in the many-body localized phase," Phys. Rev. B 91, 085425 (2015).

[24] D. J. Luitz, N. Laflorencie, and F. Alet, "Many-body localization edge in the random-field Heisenberg chain," Phys. Rev. B 91, 081103 (2015).

[25] Y. BarLev, G. Cohen, and D. R. Reichman, "Absence of diffusion in an interacting system of spinless fermions on a one-dimensional disordered lattice," Phys. Rev. Lett. 114, 100601 (2015).

[26] R. Mondaini and M. Rigol, "Many-body localization and thermalization in disordered Hubbard chains," Phys. Rev. A 92, 041601 (2015).

[27] R. Vasseur, S. A. Parameswaran, and J. E. Moore, 
"Quantum revivals and many-body localization," Phys. Rev. B 91, 140202 (2015).

[28] S. Bera, H. Schomerus, F. Heidrich-Meisner, and J. H. Bardarson, "Many-body localization characterized from a one-particle perspective," Phys. Rev. Lett. 115, 046603 (2015).

[29] G. Carleo, F. Becca, M. Schiró, and M. Fabrizio, "Localization and glassy dynamics of many-body quantum systems," Scientific Reports 2, 243 (2012).

[30] T. Grover and M. P. A. Fisher, "Quantum disentangled liquids," J. Stat. Mech.: Theory and Experiment , P10010 (2014).

[31] W. De Roeck and F. Huveneers, "Asymptotic quantum many-body localization from thermal disorder," Comm. in Math. Phys. 332, 1017 (2014).

[32] M. Schiulaz and M. Müller, "Ideal quantum glass transitions: Many-body localization without quenched disorder," AIP Conf. Proc. 1610, 11 (2014).

[33] W. De Roeck and F. Huveneers, "Can translation invariant systems exhibit a many-body localized phase?" arXiv preprint arXiv:1409.8054 (2014).

[34] M. Schiulaz, A. Silva, and M. Müller, "Dynamics in many-body localized quantum systems without disorder," Phys. Rev. B 91, 184202 (2015).

[35] L. Barbiero, C. Menotti, A. Recati, and L. Santos, "Outof-equilibrium states and quasi-many-body localization in polar lattice gases," Phys. Rev. B 92, 180406 (2015).

[36] M. van Horssen, E. Levi, and J. P. Garrahan, "Dynamics of many-body localization in a translation-invariant quantum glass model," Phys. Rev. B 92, 100305 (2015).

[37] Z. Papič, E. M. Stoudenmire, and D. A. Abanin, "Manybody localization in disorder-free systems: The importance of finite-size constraints," Ann. of Phys. 362, 714 (2015).

[38] I. H. Kim and J. Haah, "Localization from superselection rules in translationally invariant systems," Phys. Rev. Lett. 116, 027202 (2016).

[39] N. Y. Yao, C. R. Laumann, J. I. Cirac, M. D. Lukin, and J. E. Moore, "Quasi-many-body localization in translation-invariant systems," Phys. Rev. Lett. 117, 240601 (2016).

[40] Y. Kagan and L. A. Maksimov, "Localization in a system of interacting particles diffusing in a regular crystal," Zh. Eksp. Teor. Fiz. 87, 348 (1984).

[41] Y. Kagan and L. A. Maksimov, "Effect of interparticle interaction on localization in a nonideal crystal with a narrow band," Zh. Eksp. Teor. Fiz. 88, 992 (1985).

[42] A. Smith, J. Knolle, D. L. Kovrizhin, and R. Moessner, "Disorder-free localization," Phys. Rev. Lett. 118, 266601 (2017).

[43] K.-K. Ni, S. Ospelkaus, M. H. G. de Miranda, A. Pe'er, B. Neyenhuis, J. J. Zirbel, S. Kotochigova, P. S. Julienne, D. S. Jin, and J. Ye, "A high phase-space-density gas of polar molecules," Science 322, 231 (2008).

[44] J. Stuhler, A. Griesmaier, T. Koch, M. Fattori, T. Pfau, S. Giovanazzi, P. Pedri, and L. Santos, "Observation of dipole-dipole interaction in a degenerate quantum gas," Phys. Rev. Lett. 95, 150406 (2005).

[45] S. Baier, M. J. Mark, D. Petter, K. Aikawa, L. Chomaz, Z. Cai, M. Baranov, P. Zoller, and F. Ferlaino, "Extended Bose-Hubbard Models with Ultracold Magnetic Atoms," Science 352, 201 (2016).

[46] R. Heidemann, U. Raitzsch, V. Bendkowsky, B. Butscher, R. Löw, and T. Pfau, "Rydberg excitation of bose- einstein condensates," Phys. Rev. Lett. 100, 033601 (2008).

[47] M. M. Valado, C. Simonelli, M. D. Hoogerland, I. Lesanovsky, J. P. Garrahan, E. Arimondo, D. Ciampini, and O. Morsch, "Experimental observation of controllable kinetic constraints in a cold atomic gas," Phys. Rev. A 93, 040701 (2016).

[48] K. Baumann, C. Guerlin, F. Brennecke, and T. Esslinger, "Dicke quantum phase transition with a superfluid gas in an optical cavity," Nature 464, 1301 (2010).

[49] H. Ritsch, P. Domokos, F. Brennecke, and T. Esslinger, "Cold atoms in cavity-generated dynamical optical potentials," Rev. Mod. Phys. 85, 553 (2013).

[50] R. Landig, L. Hruby, N. Dogra, M. Landini, R. Mottl, T. Donner, and T. Esslinger, "Quantum phases from competing short- and long-range interactions in an optical lattice," Nature 532, 476 (2016).

[51] R. Islam, C. Senko, W. C. Campbell, S. Korenblit, J. Smith, A. Lee, E. E. Edwards, C.-C. J. Wang, J. K. Freericks, and C. Monroe, "Emergence and frustration of magnetism with variable-range interactions in a quantum simulator," Science 340, 583 (2013).

[52] Ch. Schneider, D. Porras, and T. Schaetz, "Experimental quantum simulations of many-body physics with trapped ions," Reports on Progress in Physics 75, 024401 (2012).

[53] E. Abrahams, P. W. Anderson, D. C. Licciardello, and T. V. Ramakrishnan, "Scaling theory of localization: Absence of quantum diffusion in two dimensions," Phys. Rev. Lett. 42, 673 (1979).

[54] N. Dogra, F. Brennecke, S. D. Huber, and T. Donner, "Phase transitions in a bose-hubbard model with cavitymediated global-range interactions," Phys. Rev. A 94, 023632 (2016).

[55] Bhuvanesh Sundar and Erich J. Mueller, "Lattice bosons with infinite-range checkerboard interactions," Phys. Rev. A 94, 033631 (2016).

[56] Y. Chen, Z. Yu, and H. Zhai, "Quantum phase transitions of the Bose-Hubbard model inside a cavity," Phys. Rev. A 93, 041601 (2016).

[57] Astrid E. Niederle, Giovanna Morigi, and Heiko Rieger, "Ultracold bosons with cavity-mediated long-range interactions: A local mean-field analysis of the phase diagram," Phys. Rev. A 94, 033607 (2016).

[58] T. Flottat, L. de Forges de Parny, F. Hébert, V. G. Rousseau, and G. G. Batrouni, "Phase diagram of bosons in a two-dimensional optical lattice with infiniterange cavity-mediated interactions," Phys. Rev. B 95, 144501 (2017).

[59] N. V. Prokof'ev, B. V. Svistunov, and I. S. Tupitsyn, "Worm" algorithm in quantum Monte Carlo simulations," Phys. Lett. A 238, 253 (1998).

[60] L. Pollet, K. Van Houcke, and S. M.A. Rombouts, "Engineering local optimality in quantum Monte Carlo algorithms," Journal of Comp. Phys." 225, 2249 - 2266 (2007).

[61] L. Pollet, J. D. Picon, H. P. Büchler, and M. Troyer, "Supersolid phase with cold polar molecules on a triangular lattice," Phys. Rev. Lett. 104, 125302 (2010).

[62] In our world-line QMC simulation, each world-line is a closed curve defined in a torus; the winding number is the net number of times the world-lines wrap around the $1 \mathrm{D}$ system.

[63] S. Aubry and G. André, "Analyticity breaking and An- 
derson localization in incommensurate lattices," Ann. Israel Phys. Soc 3, 18 (1980).

[64] Y. Y. Atas, E. Bogomolny, O. Giraud, and G. Roux, "Distribution of the ratio of consecutive level spacings in random matrix ensembles," Phys. Rev. Lett. 110, 084101 (2013).

[65] A. Angelone, F. Mezzacapo, and G. Pupillo, "Superglass phase of interaction-blockaded gases on a triangular lattice," Phys. Rev. Lett. 116, 135303 (2016).

[66] R. Díaz-Méndez, F. Mezzacapo, W. Lechner, F. Cinti, E. Babaev, and G. Pupillo, "Glass transitions in monodisperse cluster-forming ensembles: Vortex matter in type-1.5 superconductors," Phys. Rev. Lett. 118, 067001 (2017).

[67] M. Srednicki, "Chaos and quantum thermalization," Phys. Rev. E 50, 888-901 (1994).

[68] J. M. Deutsch, "Quantum statistical mechanics in a closed system," Phys. Rev. A 43, 2046-2049 (1991).

[69] M. Srednicki, "The approach to thermal equilibrium in quantized chaotic systems," Journal of Physics A: Mathematical and General 32, 1163 (1999).

[70] O. Bohigas, M. J. Giannoni, and C. Schmit, "Characterization of chaotic quantum spectra and universality of level fluctuation laws," Phys. Rev. Lett. 52, 1-4 (1984).

[71] T. A. Brody, J. Flores, J. B. French, P. A. Mello, A. Pandey, and S. S. M. Wong, "Random-matrix physics: spectrum and strength fluctuations," Rev. Mod. Phys. 53, 385 (1981).

[72] F. Haake, Quantum Signatures of Chaos (SpringerVerlag, Berlin, 1991).

[73] Despite having long-range interactions, the Hamiltonian still possesses at most two-body terms. Thus, one only expects to see full level repulsion for eigenenergies away from the ends of the spectrum [77, 78].

[74] M. Rigol, V. Dunjko, and M. Olshanii, "Thermalization and its mechanism for generic isolated quantum systems," Nature 452, 854 (2008).

[75] To the best of our knowledge, the Hamiltonian is still non-integrable even when approaching the atomic limit $(V / J \rightarrow \infty)$ for the case of incommensurate long-range interactions.

[76] A proper finite-size scaling is even more elusive here in the case of finite energy densities given that the Hilbert spaces grow exponentially with the system size, which poses a challenge to exact diagonalization studies.

[77] L. F. Santos and M. Rigol, "Onset of quantum chaos in one-dimensional bosonic and fermionic systems and its relation to thermalization," Phys. Rev. E 81, 036206 (2010).

[78] L. F. Santos and M. Rigol, "Localization and the effects of symmetries in the thermalization properties of onedimensional quantum systems," Phys. Rev. E 82, 031130 (2010). 